\title{
Secure and Practical Tree-Structure Signature Schemes Based on Discrete Logarithms
}

\author{
X.Y. Wang ${ }^{12}$, L.C. Hui ${ }^{1}$, K.P. Chow ${ }^{1}$, W.W. Tsang ${ }^{1}$, C.F. Chong ${ }^{1}$, and \\ H.W. Chan ${ }^{1}$ \\ 1 Department of Computer Science and Information Systems \\ The University of Hong Kong \\ Pokfulam, Hong Kong \\ 2 Department of Mathematics \\ Shandong University \\ Jinan 250100 PRC \\ hui@csis.hku.hk
}

\begin{abstract}
In this paper, we present another tree-structure signature scheme based on discrete logarithm problem modulo $p$, where $p$ is a large prime. The basic signing algorithm is the original ELGmal signature scheme. The scheme attains ideal security, i.e, finding existential forgeries under adaptively chosen message attacks is equivalent to solving the discrete logarithm of any random integer $y \in Z_{p}^{*}$. The scheme is also efficient, it can be implemented almost as efficiently as the original ELGamal signature scheme. We can regard the scheme as an application of ELGamal signature scheme in tree-structure signature schemes.
\end{abstract}

\section{Introduction}

Digital signature schemes are usually based on difficult number-theoretic problems. For example, the original RSA signature scheme [20] is based on the difficulty of integer factorization, and the ElGamal signature scheme [7] is based on the difficulty of discrete logarithms. The factoring problem and discrete logarithm problem are two important problems widely used to construct various signature schemes, for example, [1,2,4,5,6,11,14,15,16,19,21].

A strong requirement for digital signature scheme is that the scheme has to be "existentially unforgeable under an adaptively chosen message attack" [11], or simply "existentially unforgeable". In this attack, the adversary (or the signature forger) gets to see a signature on any message of its choice, in an adaptive manner. The forger has then to produce a signature on one message that was not previously signed, without the cooperation of the signature algorithm. A signature scheme is existentially forgeable if it cannot prevent the forger in forging a signature. On the other hand, a signature scheme is existentially unforgeable if it can be proved that the scheme prevents forging a signature in the abovementioned manner.

Many signature schemes are not existentially unforgeable. For example, both the ElGamal scheme and the RSA scheme are known to be existentially forgeable. 
One practical solution to this problem is to use those schemes together with some secure hash function. That is, the message to be signed has to be processed by a hash function first, and then the hash value is signed. While this approach works fine in practice, in theory this approach has a drawback that the security of the overall signature scheme has to be depending on the security of the hash function as well. Therefore, it is interesting to design signature schemes that are existentially unforgeable even if no hash functions are used.

One approach to design existentially unforgeable signature schemes is to use a tree structure $[4,5,6,11,13]$. In a tree-structure signature scheme, a digital signature corresponds to a path of a specially designed tree, from the root to a leaf. Each edge in the path corresponds to some "basic" digital signature technique, for example RSA. A signature in a tree-structure signature scheme can be viewed as a sequence of signatures in the basic scheme together with some extra information.

Early tree-structure signatures usually have larger signature sizes than similar signatures directly based on the respective basic problems. For example, the size of [13] is $O\left(l^{2} \log i\right)$.

[11] reduces the size to $O(l d)$, where $d$ is the depth of a binary tree used in the signature scheme, and $l$ is a security parameter which often indicates the length of the main input, for example, in [11], $l$ is the binary length of the modulo $n=p q$. [5] and [6] also have small signature size of $O(l d)$, here $d$ also is the depth of the tree and not more than 3. This is only a constant times the signature size of the basic algorithm, which is the original RSA signature scheme [20]. Tree-structure signature schemes having this efficiency property are known as "practical" schemes.

In the security aspect of [11], there are no existential forgery under the assumption that the factoring problem is difficult to solve. This means that if somebody can forge a signature of [11], then there is a probabilistic polynomialtime attack with non-negligible probability to factor the modulo $n$. In this paper, we denote the scheme as achieving "ideal" security under the factorization assumption. In the security aspect of [5] and [6], two schemes achieve the "ideal" security under the RSA assumption.

[4] describes a general method for constructing secure tree-structure signature schemes based on interactive protocols. In addition, the paper introduces an example of applying the general method to obtain a secure signature scheme based on the discrete logarithm problem. The scheme achieves the "ideal" security under the discrete logarithm assumption.

In this paper, we propose an alternate tree-structure signature scheme also based on discrete logarithms. Our scheme uses the original ElGamal signature [7] as it's basic signing algorithm. The scheme attains ideal security under the discrete logarithm assumption, i.e, finding existential forgeries under adaptively chosen message attacks is equivalent to solving the discrete logarithm of any random integer $y \in Z_{p}^{*}$. The scheme has similar efficiency as [4]. It outputs a signature with a signature size about $2 d$ times that of the original ElGamal signature size, here $d$ is the depth of the binary tree used in the scheme. For 
example, If the depth of the tree is 32 , the signer at most can generate $2^{32}$ signatures, then the signature size is 64 times that of the original ELGamal signature. Therefore our scheme is also practical.

The organization of the rest of the paper is as follows. Section 2 gives an overview of tree-structure signature schemes. Section 3.1 describes the details of the scheme, and sections 3.2 and 3.3 analyse the efficiency and security of the scheme, respectively. Finally in section 4, the paper is concluded.

\section{Tree-Structure Signatures}

The tree structure in this paper is a binary tree which is similar to that of the tree-structure signature scheme in [11]. $d$ represents the depth of the tree. A signer can make at most $2^{d}$ signatures.

A value is associated with each internal node. We denote the value in the root by $Y_{0}=\left(x_{0}, y_{01}, y_{02}\right)$. The signing process starts from the leftmost unused path. The value in the root is a part of the public key. The values in all internal nodes are progressively defined with the signing process by the signer. For example, If the signer generates the $i$-th signature, all the internal nodes appeared in the previous $(i-1)$ signatures will have their values defined.

Assume that the $i$-th path is $\left(i_{1}, i_{2}, \ldots i_{d}\right)$ which has the same first $j$ internal nodes with $(i-1)$-th path, then in the $i$-th path, the first $j$ nodes have their values defined coincide with those of the $(i-1)$-th signature. The remaining $d-j$ nodes will be given their new values according to the signing algorithm. All the nodes that do not appear in first $i$ signatures will not have their values defined. After $2^{d}$ different signatures have been generated, any node of the tree employs a fixed value.

In the signature scheme presented in this paper, each internal node associates with a sequence of 3 elements. The choice of the value corresponding to each leaf is different from the values of internal nodes. The value of each leaf is a sequence containing two elements.

In addition, we provide that if the signer generates the $i$-th signature, he must store the $(i-1)$-th signature in advance. This is a property of tree-structure signatures. Certainly, the scheme can be made totally "memoryless" like [11].

\section{Signature Schemes Based on Discrete Logarithm Problem}

\subsection{The Description of the Signature Scheme}

Key generation: The signer selects a big prime $p$ such that $p-1=a q, q$ is a large prime and $a$ is a small factor. Select a generator $g \in Z_{p}^{*}$. Select $g_{1} \equiv$ $g^{\gamma_{1}} \bmod p, g_{2} \equiv g^{\gamma_{2}} \bmod p, x_{0} \equiv g^{\alpha_{0}} \bmod p, y_{0 t} \equiv g^{u_{0 t}} \bmod p, t=1,2$ such that $g . c . d\left(\alpha_{0}, p-1\right)=1, \operatorname{g.c} . d\left(\gamma_{t}, p-1\right)=1, \operatorname{g.c} . d\left(u_{0 t}, p-1\right)=1, t=1,2$, and $\alpha_{0}, \gamma_{t}, u_{0 t}$ are random integers in $Z_{p-1}^{*}$. 


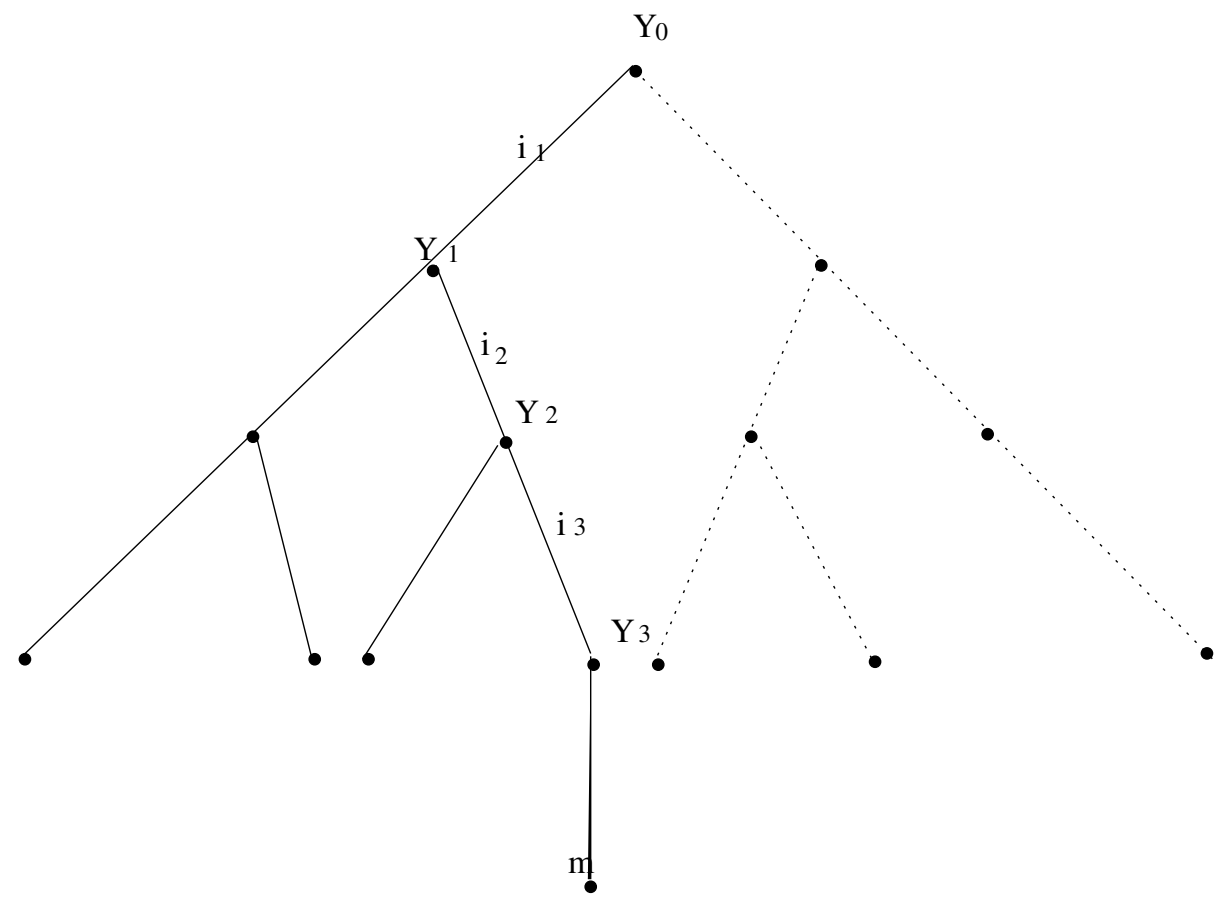

Fig. 1. The Tree with $d=3$

Public key: The public key is $\left(p, Y_{0}, g, g_{1}, g_{2}\right)$, where $Y_{0}$ is a sequence including 3 elements $Y_{0}=\left(x_{0}, y_{01}, y_{02}\right)$.

Private key: The private key is $\left(\alpha_{0}, \gamma_{1}, \gamma_{2}, u_{01}, u_{02}\right)$.

Signing: In the tree of the scheme, each node will be associated with a sequence of 3 elements. We denote the sequence as $Y_{j}=\left(x_{j}, y_{j 1}, y_{j 2}\right)$, where $x_{j}$, $y_{j 1}, y_{j 2}$ are selected randomly by the signer, index $x_{p, g}\left(x_{j}\right)$, index $x_{p, g}\left(y_{j t}\right), t=1,2$ are kept secret by the signer until the next signature is generated. index $x_{p, g}\left(x_{j}\right)$ denotes the discrete logarithm of $x_{j}$ about the modulo $p$ and the base $g$.

The signing can be described as follows (see Figure 1):

Step 1: The signer chooses the i-th path $\left(i_{1}, i_{2}, \ldots i_{d}\right)$ of the tree for the $i$-th signature. If the nodes $i_{1}, i_{2}, \ldots i_{j}$ have their corresponding sequences defined in the first $(i-1)$ signatures, let

$$
\begin{gathered}
Y_{k} \longleftarrow Y_{k}, k=1,2, \ldots j \\
s_{k} \longleftarrow s_{k}, \quad r_{k} \longleftarrow r_{k}, \quad t_{k} \longleftarrow t_{k}, k=1,2, \ldots j
\end{gathered}
$$

Otherwise choose random $\alpha_{k}, u_{k t} \in Z_{p-1}^{*}, k=j+1, \ldots d-1, t=1,2$, where g.c.d $\left(\alpha_{k}, p-1\right)=1$ and $g . c . d\left(u_{k t}, p-1\right)=1$, and let

$$
\begin{aligned}
& x_{k} \longleftarrow g^{\alpha_{k}} \bmod p \\
& y_{k t} \longleftarrow g^{u_{k t}} \bmod p
\end{aligned}
$$




$$
Y_{k} \longleftarrow\left(x_{k}, y_{k 1}, y_{k 2}\right)
$$

Step 2: For $k=d$, choose random $\alpha_{d}, u_{d 1} \in Z_{p-1}^{*}$ such that g.c.d $\left(\alpha_{d}, p-1\right)=$ 1 , and $\operatorname{g.c.d}\left(u_{d 1}, p-1\right)=1$. Let

$$
\begin{gathered}
x_{d} \longleftarrow g^{\alpha_{d}} \bmod p \\
y_{d 1} \longleftarrow g^{u_{d 1}} \bmod p \\
Y_{d} \longleftarrow\left(x_{d}, y_{d 1}\right)
\end{gathered}
$$

Step 3: For $k=j+1, \ldots d$, if $x_{k}$ is the $i_{k}$-th child of its parent, here $i_{k}=1$ or 2. Compute $s_{k}, t_{k}, r_{k}$ such that:

$$
\begin{aligned}
g^{x_{k}} & \equiv x_{k-1}^{s_{k}} \cdot\left(y_{k-1, i_{k}}\right)^{x_{k-1}} \bmod p \\
& g_{1}^{y_{k 1}} g_{2}^{y_{k 2}} \equiv x_{k}^{t_{k}} \cdot x_{0}^{r_{k}} \cdot g^{x_{k}} \bmod p
\end{aligned}
$$

If $k=d$, let $y_{d 2}=0$.

This is equivalent to computing $s_{k}, t_{k}, r_{k}$ such that:

$$
\begin{gathered}
x_{k} \equiv \alpha_{k-1} \cdot s_{k}+u_{k-1, i_{k}} \cdot x_{k-1} \quad \bmod (p-1) \\
\gamma_{1} \cdot y_{k 1}+\gamma_{2} \cdot y_{k 2} \equiv \alpha_{k} \cdot t_{k}+\alpha_{0} \cdot r_{k}+x_{k} \bmod (p-1)
\end{gathered}
$$

(Note: The second equation has many solutions, the signer randomly selects one.)

Step 4: For the message $m \in[0, q-1]$, compute

$$
g^{m} \equiv x_{d}^{s} \cdot\left(y_{d 1}\right)^{x_{d}} \bmod p
$$

Output: The signature of message $m$ is:

$$
S(m)=\left(Y_{1}, i_{1}, s_{1}, t_{1}, r_{1} ; \ldots . Y_{d}, i_{d}, s_{d}, t_{d}, r_{d} ; m, s\right)
$$

here $Y_{d}=\left(x_{d}, y_{d 1}\right)$.

Verification: Given the signature $S(m)$, the verification of a signature is as follows:

$$
S(m)=\left(Y_{1}, i_{1}, s_{1}, t_{1}, r_{1} ; \ldots . Y_{d}, i_{d}, s_{d}, t_{d}, r_{d} ; m, s\right)
$$

The receiver verifies that:

$$
\begin{gathered}
g^{x_{k}} \equiv x_{k-1}^{s_{k}} \cdot\left(y_{k-1, i_{k}}\right)^{x_{k-1}} \bmod p, k=1,2 \\
g_{1}^{y_{k 1}} \cdot g_{2}^{y_{k 2}} \equiv x_{k}{ }^{t_{k}} \cdot x_{0}^{r_{k}} \cdot g^{x_{k}} \bmod p \\
g^{m} \equiv x_{d}^{s} \cdot\left(y_{d}^{x_{d}}\right) \bmod p
\end{gathered}
$$

It is noted that, in the $i$-th signature, the sequences corresponding to all the nodes are the following:

$$
\left(x_{1}, y_{11}, y_{12}\right), \ldots .\left(x_{d-1}, y_{d-1,1}, y_{d-1,2}\right),\left(x_{d}, y_{d 1}\right)
$$


$s_{k}$ is equivalent to the signature of $x_{k}$, and $\left(t_{k}, r_{k}\right)$ is the common signature of $y_{k 1}$ and $y_{k 2}, \mathrm{k}=1,2, \ldots \mathrm{d}$. The signature $S(m)$ in fact includes all the signatures of all the elements appeared in the path.

We provide that if $S(m)$ and $S\left(m^{\prime}\right)$ are two different signatures of $i$-th path, $S(m)$ must at least include a parameter pair $\left(x_{k}, y_{k 1}, y_{k 2}\right)$ different from the corresponding pair $\left(x_{k}^{\prime}, y_{k 1}^{\prime}, y_{k 2}^{\prime}\right)$ of $S\left(m^{\prime}\right)$ or $\left(x_{k}, y_{k 1}, y_{k 2}\right)=\left(x_{k}^{\prime}, y_{k 1}^{\prime}, y_{k 2}^{\prime}\right), k=$ $1,2, \ldots d$, but $m \neq m^{\prime}$.

\subsection{Performance of the Signature Scheme}

A signer can use the tree to make at most $2^{d}$ signatures. The signature size is about $O(l d)$ bits. the public key size is about $O(l)$ bits.

When the signer generates the $i$-th signature, he must store the $(i-1)$-th, $i$-th signatures and the discrete logarithms of $x_{k}, y_{k t}, t=1,2$ appears in two signatures. So the storage size including the secret key is at most $O(l d)$ bits which is about a few times that of the signature size.

If the signer use a pseudorandom generator to produce pseudorandom numbers as the discrete logarithms of $x_{k}, y_{k t}$, the scheme is no "memory", i.e. the signer can makes the $i$-th signature without storing any information about the $i-1$-th signature. The details can refer to [10].

The verification is almost as efficient as the original signature scheme, and the execution time is about $d$ times that of the original ElGamal signature verification.

\subsection{The Proof of the Security about Signature Scheme}

In this section, we show that the signature scheme has no existential forgery under adaptively chosen message attacks, using the simulated signature concept used in $[5,6,11]$. The proof is divided into two parts. In the first part (Theorem 1 ), we demonstrate that there is a simulator that can simulate the signing process by selecting another public key $Y_{0}$ where discrete logarithm of each element in $Y_{0}$ is unknown. The simulated signatures and the real signatures are indistinguishable. This demonstrates that our design is zero-knowledge, i.e, the real signatures cannot leave any information about secret keys. The second part (Theorem 2) shows that, if an attacker can forge a signature of the real signature scheme under an adaptively chosen message attack with a non-negligible probability, the attacker can find a probabilistic polynomial algorithm to compute the discrete logarithm of any random integer $y$ in $Z_{p}^{*}$. So, our signature scheme achieves the ideal security.

Theorem 1 There is a simulator which can simulate the signing process, i.e, the simulator can select another root sequence $Y_{0}=\left(x_{0}, y_{01}, y_{02}\right)$, where inde $_{p, g}\left(x_{0}\right)$ and $i n d e x_{p, g}\left(y_{0 t}\right), t=1,2$ are all unknown. The simulating process and the real signing process are indistinguishable in polynomial time.

Proof: The simulator first chooses $Y_{0}=\left(x_{0}, y_{01}, y_{02}\right)$ as follows:

Select any random integer $y \in Z_{p}^{*}$, random integer $\alpha_{0}, \beta_{0} \in Z_{p-1}^{*}$, Let $x_{0} \longleftarrow$ $g^{\alpha_{0}} \cdot y^{\beta_{0}}$, and provide that $\operatorname{g.c} . d\left(x_{0}, p-1\right)=1, \operatorname{g.c} . d\left(\beta_{0}, p-1\right)=1$. 
Select random integer $\gamma_{1}, \gamma_{2}, \delta_{1}, \delta_{2} \in Z_{p-1}^{*}$ such that:

$$
\begin{aligned}
& g_{1} \longleftarrow g^{\gamma_{1}} \cdot y^{\delta_{1}} \\
& g_{2} \longleftarrow g^{\gamma_{2}} \cdot y^{\delta_{2}}
\end{aligned}
$$

Select first elements of $Y_{11}, Y_{12}$ which are two children of $Y_{0}: x_{1 t} \equiv g^{\alpha_{1 t}} \cdot y^{\beta_{1 t}}$, $t=1,2$ such that $\alpha_{0} \cdot \beta_{1 t}-\beta_{0} \cdot \alpha_{1 t} \neq 0 \bmod p-1$, and $\alpha_{1 t}$ and $\beta_{1 t}$ are random integers in $Z_{p-1}^{*}$. Corresponding to $x_{1 t}$, select random integer $s_{1 t} \in Z_{p-1}$.

After $x_{1 t}, s_{1 t}, t=1,2$ are selected, we can select $y_{0 t}, t=1,2$. Assume that: $y_{0 t}=g^{u_{0 t}} \cdot y^{v_{0 t}} \bmod p, t=1,2$, here $u_{0 t}, v_{0 t}, t=1,2$ are unknown parameters.

Compute $y_{0 t}$ satisfies the following equation:

$$
g^{x_{1 t}} \equiv x_{0}^{s_{1 t}} \cdot y_{0 t}^{x_{0}} \bmod p
$$

So,

$$
g^{x_{1 t}} \equiv g^{\left(\alpha_{0} . s_{1 t}+u_{0 t} \cdot x_{0}\right)} \cdot y^{\beta_{0} \cdot s_{1 t}+v_{0 t} \cdot x_{0}}
$$

¿From g.c.d $\left(x_{0}, p-1\right)=1$, we know that the equation has one solution. We determine $u_{0 t}$ and $v_{0 t}$ as the only solution of the system equations:

$$
\left\{\begin{array}{c}
\alpha_{0} \cdot s_{1 t}+u_{0 t} \cdot x_{0} \equiv x_{1 t} \quad \bmod p-1 \\
\beta_{0} \cdot s_{1 t}+v_{0 t} \cdot x_{0} \equiv 0 \quad \bmod p-1
\end{array}\right.
$$

Similar to the above method, if the first element $x_{k}$ of $Y_{k}=\left(x_{k}, y_{k 1}, y_{k 2}\right)$ is defined, the other elements $y_{k 1}$ and $y_{k 2}$ will be defined after two first elements $x_{k+1,1}$ and $x_{k+1,2}$ of $Y_{k+1,1}$ and $Y_{k+1,2}$ defined where $Y_{k 1}$ and $Y_{k 2}$ are two children of $Y_{k}$.

If the simulator has output $(i-1)$ simulated signatures, for any unsigned message $\mathrm{m}$, the $i$-th signatures $S(m)$ is generated as follows:

The simulator chooses the $i$-th path $\left(i_{1}, i_{2}, \ldots i_{l}\right)$ of the tree. If the path has $j-1$ common internal nodes with the $(i-1)$-th signature, the first $j-1$ nodes of the $i$-th path has the same sequences $\left(Y_{1}, Y_{2}, \ldots Y_{j-1}\right)$ with the former signature. The sequence corresponding to $j$-th node only has $x_{j}$ defined, and $y_{j t}, t=1,2$ are not defined. Similar to the choices of $x_{1 t}, s_{1 t}$ and $y_{0 t}, t=1,2$, the simulator choose $x_{j+1, t}, s_{j+1, t}, y_{j t}, t=1,2$ where $x_{j+1, t} \equiv g^{\alpha_{j+1, t}} \cdot y^{\beta_{j+1, t}}$, $y_{j t} \equiv g^{u_{j t}} \cdot y^{v_{j t}} \bmod p, t=1,2$ such that:

$$
\begin{gathered}
g . c . d\left(x_{j+1, t}, p-1\right)=1 \\
\alpha_{0} \cdot \beta_{(j+1) t}-\beta_{0} \cdot \alpha_{(j+1) t} \neq 0 \quad \bmod p-1, \\
g^{x_{(j+1) t}} \equiv x_{j}^{s_{(j+1) t}} \cdot y_{j, i_{j+1}}^{x_{j}} \quad\left(2^{\prime}\right)
\end{gathered}
$$

After $x_{k}, y_{k t}, s_{k}, k=1,2, \ldots d, t-1,2$ are simulated, $t_{k}, r_{k}$ are computed as follows:

$$
g_{1}^{y_{k 1}} g_{2}^{y_{k 2}} \equiv x_{k}^{t_{k}} \cdot x_{0}^{r_{k}} \cdot g^{x_{k}} \bmod p
$$


So,

$$
g^{\gamma_{1} \cdot y_{k 1}+\gamma_{2} \cdot y_{k 2}} \cdot y^{\delta_{1} \cdot y_{k 1}+\delta_{2} \cdot y_{k 2}} \equiv g^{\alpha_{k} \cdot t_{k}+\alpha_{0} \cdot r_{k}+x_{k}} \cdot y^{\beta_{k} \cdot t_{k}+\beta_{0} \cdot r_{k}} \bmod p
$$

Compute $t_{k}, r_{k}$ satisfy the following system equations:

$$
\left\{\begin{array}{c}
\alpha_{k} \cdot t_{k}+\alpha_{0} \cdot r_{k}+x_{k} \equiv \gamma_{1} \cdot y_{k 1}+\gamma_{2} \cdot y_{k 2} \bmod p-1 \\
\beta_{k} \cdot t_{k}+\beta_{0} \cdot r_{k} \equiv \delta_{1} \cdot y_{k 1}+\delta_{2} \cdot y_{k 2} \bmod p-1
\end{array}\right.
$$

¿From $\alpha_{k} \cdot \beta_{0}-\alpha_{0} \cdot \beta_{k} \neq 0 \bmod p-1$, the system equations has one solution. After $x_{d}, s$ are selected, the simulator computes $y_{d 1}$ such that:

$$
g^{m} \equiv x_{d}^{s} \cdot\left(y_{d 1}\right)^{x_{d}} \quad \bmod p
$$

The $i$-th simulated signature is:

$$
S(m)=\left(Y_{1}, i_{1}, s_{1}, t_{1}, r_{1} ; \ldots . . Y_{d}, i_{d}, s_{d}, t_{d}, r_{d} ; m, s\right)
$$

So, the simulator runs the whole simulating process by the above method. The simulating process is indistinguishable from a real signing process in polynomial time. Because in the real signature scheme, the signer selects $x_{k}, x_{k-1}, y_{k-1, i_{k}}$ which are indistinguishable from random integers in $Z_{p}^{*} . s_{k}$ is the only solution of the equation $\left(1^{\prime}\right)$. In the simulated signature scheme, the simulator selects $x_{k}, x_{k-1}, s_{k}$ which are indistinguishable from random integer in $Z_{p}^{*}, y_{k-1, i_{k}}$ is the only solution of equation $\left(2^{\prime}\right)$. So, all the parameters $x_{k}, x_{k-1}, s_{k}, y_{k-1, i_{k}}$ in the simulated signature are indistinguishable from the corresponding parameters in the real signature. Because anyone besides the simulator don't know $y$, inde $x_{p, g}\left(g_{1}\right)$, index $x_{p, g}\left(g_{2}\right)$, inde $x_{p, g}\left(x_{k}\right)$, inde $x_{p, g}\left(x_{0}\right)$, he cannot distinguish $t_{j}, r_{j}$ like the simulator, so $t_{j}, r_{j}$ in the simulated signature are indistinguishable from that of the real signature. Therefore these two schemes are indistinguishable.

Theorem 2: If there is an algorithm that can forge a signature under adaptively chosen message attacks with a non-negligible probability, there is a probabilistic polynomial-time algorithm $A$ to compute the discrete logarithm problem, i.e. given any random integer $y \in Z_{p}^{*}, A$ can output index $x_{p, g}(y)$ with a non-negligible probability.

Proof: Select any random integer $y \in Z_{p}^{*}$, We run the above entire simulated process. If an algorithm can forge a legitimate signature of the real signing scheme under adaptively chosen message attacks with an non-negligible probability, the algorithm can forge a legitimate signature of the simulated signature scheme with the same probability.

We provide that, after $2^{d}$ calls to the simulation, the attack algorithm forges a new signature different from the simulated signatures.

$$
S\left(m^{\prime}\right)=\left(Y_{1}^{\prime}, i_{1}, s_{1}^{\prime}, t_{1}^{\prime} ; \ldots . . Y_{d}^{\prime}, i_{d}, s_{d}^{\prime}, t_{d}^{\prime} ; m^{\prime}, s^{\prime}\right)
$$

For the same path, the simulated signature is:

$$
S(m)=\left(Y_{1}, i_{1}, s_{1}, t_{1} ; \ldots . . Y_{d}, i_{d}, s_{d}, t_{d} ; m, s\right)
$$


Provided that $S(m)$ and $S\left(m^{\prime}\right)$ have $(j-1)$ internal nodes that have the same pairs $\left(x_{k}, y_{k 1}, y_{k 2}\right), k=1,2$. Because the root is fixed, $j$ must exist. For the $j$-node, $\left(x_{j}, y_{k 1}, y_{k 2}\right) \neq\left(x_{j}^{\prime}, y_{k 1}^{\prime}, y_{k 2}^{\prime}\right)$.

We prove the following 3 cases:

Case 1: If $x_{j} \neq x_{j}^{\prime}$, We get the following two equation:

$$
g^{x_{j}^{\prime}} \equiv x_{j-1}^{s_{j}^{\prime}} \cdot\left(y_{j-1, i_{j}}\right)^{x_{j-1}} \bmod p
$$

So,

$$
g^{x_{j}^{\prime}} \equiv g^{\alpha_{j-1} \cdot s_{j}^{\prime}+u_{j-1, i_{j}} \cdot x_{j-1}} \cdot y^{\beta_{j-1} \cdot s_{j}^{\prime}+v_{j-1, i_{j}} \cdot x_{j-1}} \bmod p
$$

We get the following equation:

$$
g^{u} \equiv y^{v} \quad \bmod p
$$

here

$$
\begin{gathered}
u \equiv x_{j}^{\prime}-\alpha_{j-1} \cdot s_{j}^{\prime}-u_{j-1, i_{j}} \cdot x_{j-1} \bmod p \\
v \equiv \beta_{j-1} \cdot s_{j}^{\prime}+v_{j-1, i_{j}} \cdot x_{j-1} \quad \bmod p
\end{gathered}
$$

Because $g . c . d\left(x_{j-1}, p-1\right)=1$, and the attacker don't know the parameters $\alpha_{j-1}, \beta_{j-1}, u_{j-1, i_{j}}, v_{j-1, i_{j}}$, so $\operatorname{g.c} . d(v, q)=1$ holds with the high probability of $1-\frac{a}{p-1}=1-\frac{1}{q}$. We can provide that $\operatorname{g.c} . d(v, q)=1$.

If $g . c . d(v, p-1)=1$, we easily compute $\operatorname{index}_{p, g}(y) \equiv u . v^{-1} \bmod (p-1)$.

If $g . c . d(v, p-1)=t>1$, t is small, we know $i_{n d e x_{p, g}}(y)$ is a solution of the following equation:

$$
u \equiv x . v \bmod p-1
$$

So, we can compute the only solution $x^{\prime}$ of the following equation:

$$
\frac{u}{t} \equiv x \cdot \frac{v}{t} \quad \bmod \quad \frac{p-1}{t}
$$

So, equation $\left(4^{\prime}\right)$ has $t$ solutions such that $x^{\prime}+i \cdot \frac{p-1}{t}, i=0,1, \ldots t-1$. We can verify which solution is $i n d e x_{p, g}(y)$. Therefore $i n d e x_{p, g}(y)$ is easily computed.

Case 2: If $x_{j}=x_{j}^{\prime}$, then $\left(y_{j 1}, y_{j 2}\right) \neq\left(y_{j 1}^{\prime}, y_{j 2}^{\prime}\right)$. So,

$$
\begin{gathered}
g_{1}^{y_{j 1}} \cdot g_{2}^{y_{j 2}} \equiv x_{j}^{t_{j}} \cdot x_{0}^{r_{j}} \cdot g^{x_{j}} \bmod p \\
g_{1}^{y_{j 1}^{\prime}} \cdot g_{2}^{y_{j 2}^{\prime}} \equiv x_{j}^{t_{j}^{\prime}} \cdot x_{0}^{r_{j}{ }^{\prime}} \cdot g^{x_{j}} \bmod p
\end{gathered}
$$

¿From $\left(6^{\prime}\right)$, we get the following equation:

$$
g^{\gamma_{1} \cdot y_{j 1}^{\prime}+\gamma_{2} \cdot y_{j 2}^{\prime}} \cdot y^{\delta_{1} \cdot y_{j 1}^{\prime}+\delta_{2} \cdot y_{j 2}^{\prime}} \equiv g^{\alpha_{j} \cdot t_{j}^{\prime}+\alpha_{0} \cdot r_{j}^{\prime}+x_{j}} \cdot y^{\beta_{j} \cdot t_{j}^{\prime}+\beta_{0} \cdot r_{j}^{\prime}} \bmod p
$$

So,the following equation holds:

$$
g^{u} \equiv y^{v} \quad \bmod p
$$


here

$$
\begin{gathered}
u \equiv \gamma_{1} \cdot y_{j 1}^{\prime}+\gamma_{2} \cdot y_{j 2}^{\prime}-\alpha_{j} \cdot t_{j}^{\prime}-\alpha_{0} \cdot r_{j}^{\prime}-x_{j} \quad \bmod p-1 \\
v \equiv-\delta_{1} \cdot y_{j 1}^{\prime}-\delta_{2} \cdot y_{j 2}^{\prime}+\beta_{j} \cdot t_{j}^{\prime}+\beta_{0} \cdot r_{j}^{\prime} \quad \bmod p-1
\end{gathered}
$$

Because g.c.d $\left(x_{j}, p-1\right)=1$, and the attacker don't know $y, \alpha_{t}, \beta_{t}, t=0,1,2$; $\gamma_{t}, \delta_{t}, t=1,2$. So, similar to case 1 , we can provided that $\operatorname{g.c} . d(u, q)=1$, then g.c.d $(v, q)=1$.

If $g . c . d(v, p-1)=1$, we can easily compute $\operatorname{index}_{p, g}(y) \equiv u v^{-1} \bmod p-1$.

If $g . c . d(v, p-1)=t$ is small,we compute $\operatorname{index}_{p, g}(y)$ similar to Case 1.

Case 3: For $j=1,2, . . d,\left(x_{j}, y_{j, i_{j+1}}\right)=\left(x_{j}^{\prime}, y_{j, i_{j+1}}^{\prime}\right)$. So, $m \neq m^{\prime}$. The following equation holds:

$$
g^{m-m^{\prime}} \equiv x_{d}^{s-s^{\prime}} \equiv\left(g^{\alpha_{d}} \cdot y^{\beta_{d}}\right)^{s-s^{\prime}} \bmod p
$$

Because $m, m^{\prime} \in[0, q-1]$, g.c.d $\left(q, m-m^{\prime}\right)=1$ holds. Similar to case 1 , we can compute index $x_{p . g}(y)$.

So, if there is an algorithm that can forge a signature under adaptively chosen message attack with a non-negligible probability, there is a probabilistic polynomial-time algorithm $A$ to compute the discrete logarithm problem with a non-negligible probability. Thus the signature scheme is no existentially forgeable under adaptively chosen message attack.

\section{Conclusion and Discussions}

In this paper, we have introduced an alternate secure and efficient tree-structure signature scheme based on discrete logarithms, in addition to the scheme introduced in [4]. The scheme is secure and efficient. It is interesting to know that, although the original ELGamal signature scheme is existentially forgeable, it can be used to construct secure tree-structure signature scheme as the RSA does. (A more detail analysis of the differences as well as the advantages/disadvantages between our scheme and that in [4] will be presented in the conference.)

Designing a secure and efficient tree-based signature scheme has the additional advantage that although the difficulty of both the discrete logarithm and RSA problems are yet unproved, discrete logarithm problem seems to be "harder". One reason is that solving the discrete logarithm of $Z_{n}^{*}$ implies solving the factorization problem in $Z_{n}^{*}$.

\section{References}

1. G. B. Agnew, R. C. Mullin, S. A. Vanstone, Improved digital signature scheme based on discrete exponentiation, Electronics Letters, Vol. 26, pp. 1024-1025, 1990. 167

2. M. Bellare, P. Rogaway, The Exact Security of Digital Signatures-How to Sign with RSA and Rabin, Advances in Cryptology-EUROCRYPT'96, pp.399-417. 167

3. J. Bos and D. Chaum, Provably Unforgeable Signatures, Pro. Advances in Cryptology-Crypto'92 proceedings, Springer Verlag, 1993, pp. 1-14. 
4. R.Cramer, I. Damgard: Secure Signature Schemes based on Interactive Protocols, Proceedings of Crypto'95, Springer Verlag, pp.329-342. 167, 168, 176

5. R. Cramer and Ivan Damgard, New Generation of Secure and Practical RSA-based Signatures, Crypto'96,pp.173-185. 167, 168, 172

6. C. Dwork, Moni Naor, An Efficient Existentially Unforgeable Signature Scheme and Its Applications, Crypto'94, pp. 234-246. 167, 168, 172

7. T. ElGamal, A Public-key Cryptosystem and a Signature Scheme based on Discrete Logarithm, IEEE Transactions on Information Theory, IT-31(4):469-472, 1985. 167,168

8. R. Gennaro, S. Halevi, T. Rabin, Secure Hash-and-Sign Signatures Without the Random Oracle, Advances in Cryptology-EUROCRYPT'99, pp.123-139.

9. R. Gennaro, H. Krawczyk, T. Rabin, RSA-based Undeniable Signature, Pro. Advances in Cryptology-Crypto'97, Springer Verlag, 1998, pp.133-148.

10. O. Goldreich, Two Remarks Concerning the Goldwasser-Micali-Rivest Signature Scheme, Proceedings of Crypto'86, pp.104-110. 172

11. S. Goldwasser, S. Micali and R. Rivest, A Digital Signature Scheme Secure against Chosen Message Attacks, SIAM Journal on Computing, 17(2):281-308, 1988. 167, 168, 169, 172

12. P. Horster, H. Petersen, M. Michels, Meta-ELGamal signature schemes, 2nd ACM Conference on Computer and Communications Security, pp.96-107, 1994.

13. R. C. Merkle, A Certified Digital Signature, Proceedings of Crypto'89, Springer Verlag LNCS series, pp.234-246. 168

14. M. Michels and M. Stadler, Generic Constructions for Secure and Efficient Confirmer Signature Schemes, Advances in Cryptology- EUROCRYPT'98, pp.406-421. 167

15. National Institute of Standards and Technology, federal Information Process. Standard, FIPS PUB 186-1: Digital Signature Standard (DSS), 1991. 167

16. K. Nyberg, R. Rueppel, message recovery for signature schemes based on the discrete logarithm problem, Eurocrypto'94, pp.182-193, 1994. 167

17. K.Ohta, T. Okamoto, On Concrete Security Treatment of Signatures Derived from Identification, Advances on Cryptology-CRYPTO'98, pp. 354-369.

18. T. Okamoto: Provably Secure and Practical Identification Schemes and Corresponding Signature Schemes, Proceedings of Crypto'92, Springer Verlag, pp.31-53.

19. D. Pointcheval, J.Stern, Secure Proofs for Signature Schemes, Advances in Cryptology-eurocrypt,96, pp.387-399. 167

20. R. Rivest, A. Shamir, L. Adleman, A Method for Obtaining Digital Signatures and Public Key Cryptosystem, Communications of ACM, 21(1978), pp. 120-126. 167, 168

21. S.M. Yen, C. S. Laih, New Digital Signature Scheme based on Discrete Logarithm, Electronics Letters, Vol. 29, No. 12, pp.1120-1121, 1993. 167 